\title{
Pain-Induced Pulsograph Changes in Patients with Primary Dysmenorrhea: A Pilot Study
}

\author{
Wan-hong Chen, ${ }^{1}$ Yan Zhao, ${ }^{1}$ Chang-chun Zeng, ${ }^{2}$ Dao-ning Zhang, ${ }^{1}$ Yan-ping Wang, \\ Ling Tang, ${ }^{4}$ Xiao-mei Zhang, ${ }^{5}$ and Tian-fang Wang ${ }^{1}$ \\ ${ }^{1}$ Department of Diagnostics of Traditional Chinese Medicine, School of Preclinical Medicine, Beijing University of Chinese Medicine, \\ Beijing 100029, China \\ ${ }^{2}$ School of Basic Medicine Sciences, Guilin Medical University, Guangxi, Guilin 541004, China \\ ${ }^{3}$ School of Acupuncture and Moxibustion, Beijing University of Chinese Medicine, Beijing 100029, China \\ ${ }^{4}$ Department of Gynecology, Dongzhimen Hospital, Beijing University of Chinese Medicine, Beijing 100700, China \\ ${ }^{5}$ School of Humanities, Beijing University of Chinese Medicine, Beijing 100029, China
}

Correspondence should be addressed to Tian-fang Wang; tianfangwang2000@163.com

Received 28 March 2015; Revised 7 August 2015; Accepted 10 August 2015

Academic Editor: Baiyun Zeng

Copyright (C) 2015 Wan-hong Chen et al. This is an open access article distributed under the Creative Commons Attribution License, which permits unrestricted use, distribution, and reproduction in any medium, provided the original work is properly cited.

\begin{abstract}
Objectives. To investigate changes in pulsograph caused by pain in primary dysmenorrhea (PD) patients. Methods. Pulsograph and pain level of PD patients were detected using electropulsograph and Visual-Analogue Scale (VAS), respectively, at four time points, 7-10 days before menstruation (T0), maximal pain during menstruation (T1), immediately after acupuncture analgesia (T2), and 30 mins after acupuncture analgesia (T3). Parameters $(t, h, w)$ and normalized time parameters $\left(t^{\prime}\right)$ of pulsograph were analyzed. Results. VAS pain scores decreased from $6.40 \pm 1.13$ at $T 1$ to $0.70 \pm 0.75$ at $T 2$ to $0.11 \pm 0.32$ at $T 3(P<0.001$ and 0.001$)$. At $T 1$, compared with those at T0,w1, h3, and $h 4$ significantly increased $(P<0.01)$, and $t 2, t 2^{\prime}, t 3^{\prime}$, and $h(\mathrm{~d})$ significantly decreased $(P<0.01,0.001,0.05$, and 0.001$)$. At $T 2$, compared with those at $T 1, t 1, w 1, w 2, h 2, h 3, t 1^{\prime}$, and $t 4^{\prime}$ significantly decreased $(P<0.05$, $0.01,0.01,0.001,0.01,0.001$, and 0.05$)$, and $h(\mathrm{~d})$ significantly increased $(P<0.001)$. There was no difference between $T 2$ and $T 3$. Conclusions. There are almost opposite changing trends in pulsographic parameters when pain occurs and when it is relieved in PD patients.
\end{abstract}

\section{Introduction}

Pulse assessment is an assessing method used in Traditional Chinese Medicine (TCM). The main site for pulse feeling is the radial artery located in the inner side of radial styloid process at wrist, also called cunkou in Chinese. The cunkou at each hand is divided into the following three portions, respectively. The guan position is in the middle part of cunkou, just near the radial styloid process, cun portion between the wrist crease and guan portion, and chi portion on the other side of guan portion. By feeling the pulse condition, TCM clinicians may know the unique TCM pattern status of the human body [1].

Diagnostics of TCM, a textbook widely used at TCM colleges and universities which is about how to examine illness and recognize syndrome in TCM, recorded that pain is often accompanied by wiry pulse, tight pulse, or tremulous pulse [2]. Clinical researcher also found that the wiry pulse is the most common pulse (62.9\%) in patients with pain like headaches and chest pain. Moreover, tight pulse, deep pulse, and thready pulse are also seen in those patients [3].

In view of the subjectivity of clinicians' pulse feeling, increasing interest has been focused on pulse apparatus where sensors are developed to acquire pulse signals. Pulse apparatuses are exploited to analyze pulse conditions [4]. Recently, an increasing number of studies on pulsograph have been done in clinical research [5-7]. However, only a few studies have focused on the pulsograph of patients suffering pain. One of the studies' results showed that there were significant differences in $h 1, h 3, h 4$, and $h 5$ of pulsograph 
during menstruation between different TCM patterns of 148 PD patients $[8,9]$.

Pain affects pulse conditions, giving rise to pulse changes. Accordingly, we designed the current research to investigate the pain-induced changes in pulsograph parameters. Primary dysmenorrhea (PD) is a common disease in young women. In view of its regular episode and quick relieving after treatment we chose PD representing pain to provide a basis for further study on pulse condition associated with pain in TCM.

\section{Methods}

2.1. Subjects. From April 2014 to May 2014, college students in Beijing University of Chinese Medicine who were aged 18 to 30 years with dysmenorrhea with pain level reaching 5 scores on a Visual-Analogue Scale (VAS) within the previous 3 regular menstrual cycles were recruited. PD patients who were diagnosed by the follow-up clinical examinations were invited to participate in the study. The diagnosis was made according to Primary Dysmenorrhea Clinical Guidelines made by Society of Obstetricians and Gynecologists of Canada (SOGC) in 2005. Accordingly, all patients had periodical pain in the lower abdomen before or during menstruation with normal pelvic examination and ultrasound [10].

Since unnatural changes of body may impact pulse [2], we excluded patients who had a history of injury or who caught a cold within the previous week, or who were complicated with any diseases of the heart, liver, kidney, and peripheral nerves, and so forth. For convenient detection, we excluded patients with physiologic variations or skin damage in left cunkou.

2.2. Observing Time Points. We observed PD patients at 4 time points: 7-10 days before menstruation ( $T 0)$, maximal pain during menstruation (T1), immediately after acupuncture analgesia (T2), and 30 mins after acupuncture analgesia (T3).

2.3. Pain Measurement. Pain intensity was assessed at T0, $T 1, T 2$, and $T 3$ by using a $10 \mathrm{~cm}$ VAS, a scale presented as a horizontal row of equidistant numbers from 0 to 10 , with ratings that ranged from "no pain" at 0 to "pain as bad as you can imagine" at 10 [11].

2.4. Pulse Apparatus and Gathering of Pulsograph. In this study pulsograph was detected by DS01-C electropulsograph where baroreceptors were developed to acquire pulse signals produced by Shanghai Daosheng Medical Limited Company. The pulse signals were converted into pulsograph through a computer system. The characteristics extraction and analysis of pulsograph were done by using the time-domain technique [12]. As shown in Figure 1, a standard pulsograph is composed of main wave, prodicrotic wave, and dicrotic wave. The main wave and prodicrotic wave correspond to systole while the dicrotic wave corresponds to diastole. The analyzed pulsographic parameters in this study were as below: time and amplitude of the peak of the main wave $(t 1, h 1)$ and gorge of main wave $(t 2, h 2)$ and peak of the prodicrotic wave $(t 3, h 3)$

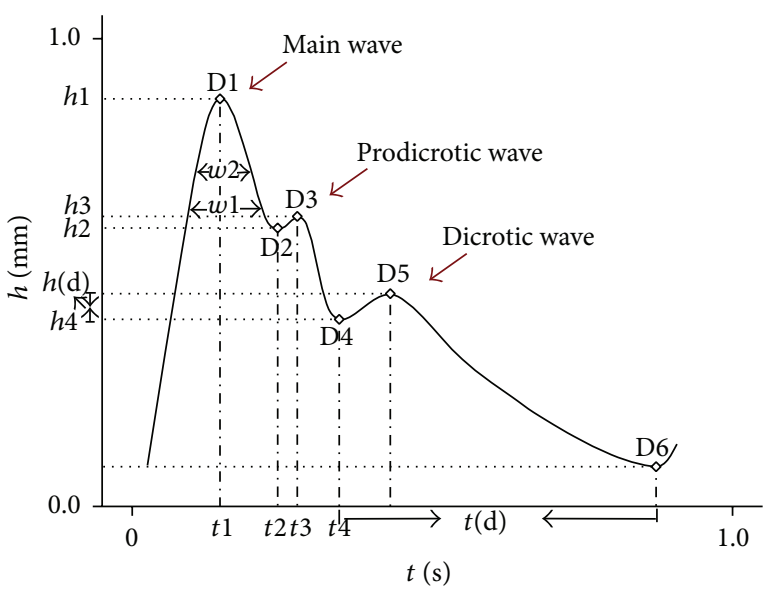

Figure 1: Pulsograph and pulsographic parameters.

and dicrotic notch $(t 4, h 4)$, width of the upper $1 / 3(w 1)$ and $1 / 5$ $(w 2)$ of the main wave, the time of dicrotic wave $(t(d))$, and amplitude of the peak of dicrotic wave $(h(\mathrm{~d}))$.

Cunkou is the main position for pulse feeling in TCM and also is the only part which electropulsograph can detect. TCM holds that left cunkou can better show the body situation of women because it mainly reflects the situation of heart, liver, and kidney. In addition, left cunkou can avoid the influence on the pulse by different motion styles in righthanded individuals. Electropulsograph only has one probe and guan position is more easily to be accurately detected. So pulsograph of guan position of left cunkou was detected in this study.

Pulsograph was detected at $T 0, T 1, T 2$, and T3. Specific detection method was as follows: patients were required in supine position, with forearm stretched, wrist straight, palm up, and wrist at the same level with heart. Trained experimenter fixed the probe of collector at the guan position of left cunkou. The computer would collect pulsographs of different pressures when the patients were relaxed and breathed naturally and then select the best pulsograph automatically for analysis.

2.5. Acupuncture Analgesia. Previous researches prove that acupuncture has instant analgesic effect [13] and is of high efficiency in primary dysmenorrhea [14]. Besides, considering the acceptance of acupuncture for subjects in this study and side effects of painkillers [15] acupuncture was chosen to alleviate primary dysmenorrhea.

Acupuncture was initiated at $T 1$. Selected acupoints included Sanyinjiao (SP6), Diji (SP8), and Yinlingquan (SP9), on both sides [16]. Manipulation is as follows: the patient was in a supine position with local skin exposed. After routine disinfection with $75 \%$ alcohol cotton balls on the local skin, disposable acupuncture needles $(40 \mathrm{~mm} \times 0.30 \mathrm{~mm})$ were then inserted at SP6, SP8, and SP9 perpendicularly in the depth of about $1 \mathrm{~cm}$. Lift, thrust, and twist gently till the patients had sensations of soreness and distension. All the needles were retained for 30 mins. 


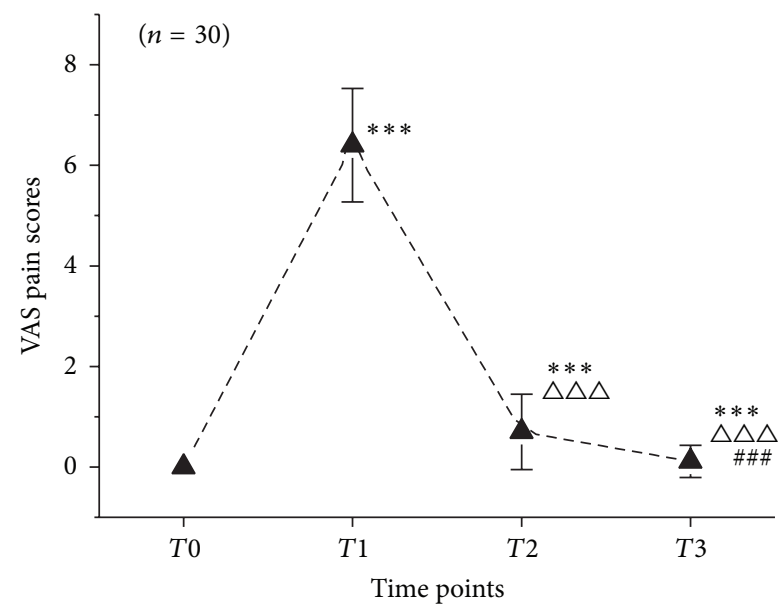

FIgURE 2: VAS pain scores of $30 \mathrm{PD}$ patients at T0, T1, T2, and T3. Notes: $T 0$ means $7-10$ days before menstruation, $T 1$ means maximal pain (VAS pain scores $\geq 5$ ) during menstruation, $T 2$ means immediately after acupuncture analgesia, and T3 means 30 mins after acupuncture analgesia. ${ }^{* * *} P<0.001$, compared with $T 0$; ${ }^{\triangle} \triangle \triangle P<0.001$, compared with $T 1 ;{ }^{\# \# \#} P<0.001$, compared with $T 2$.

2.6. Statistical Analysis. Measurement data were presented as mean \pm standard. The measurement data were analyzed by $t$ test. Differences with $P<0.05$ were considered statistically significant. The statistical evaluation was performed by using the statistical software package SPSS 17.0.

\section{Results}

3.1. Study Population. 30 patients with $\mathrm{PD}$ were enrolled with a mean age of 23 years (range, 19 to 29), and with an average suffering time of 7 years (range, 2 to 15). All the patients had provided written informed consent before completion of the experiment between May and August 2014.

3.2. VAS Pain Scores. As shown in Figure 2, 30 enrolled PD patients showed no pain at $T 0$. At $T 1$, patients experienced the maximal pain, and the reading on the VAS increased drastically to $6.40 \pm 1.13$. With acupuncture analgesia, compared with the reading at $T 1$, VAS pain scores significantly decreased to $0.70 \pm 0.75$ at $T 2(P<0.001)$. After acupuncture analgesia, the pain scores at T3 significantly decreased to $0.11 \pm 0.32(P<0.001)$, compared with that at $T 2$.

3.3. Changes in Pulsograph Parameters during Systole in PD Patients. From $T 0$ to $T 1$, time of peak of the main wave $(t 1)$ increased $(P>0.05)$ and amplitude of peak of main wave $(h 1)$ decreased $(P>0.05)$ (Figure 3(a)). Time of gorge of the main wave $(t 2)$ decreased significantly $(P<0.05)$ and amplitude of gorge of the main wave $(h 2)$ increased $(P>0.05)$ (Figure 3(b)). Time of peak of the prodicrotic wave $(t 3)$ decreased $(P>0.05)$ and amplitude of peak of prodicrotic wave $(h 3)$ increased significantly $(P<0.01)$ (Figure $3(\mathrm{c}))$. Time of dicrotic notch $(t 4)$ increased $(P>0.05)$ and amplitude of dicrotic notch $(h 4)$ increased significantly
$(P<0.01)$ (Figure 3(d)). Widths of the upper $1 / 3(w 1)$ and $1 / 5$ $(w 2)$ of main wave both increased $(P<0.01$ and $P>0.05)$ (Figure 3(e)).

From $T 1$ to $T 2$ to $T 3, t 1$ decreased gradually and there was statistically significant difference between $T 1$ and $T 2(P<$ $0.05)$. Meanwhile, $h 1$ increased gradually and was significantly higher at $T 3$ than at $T 1(P<0.05)$ (Figure 3(a)). $t 2$ increased $(P>0.05)$, while $h 2$ significantly decreased $(P<$ $0.05)$ at first and then increased $(P>0.05)$ (Figure $3(\mathrm{~b}))$. $t 3$ increased at first $(P>0.05)$ and then decreased $(P>0.05)$, while $h 3$ significantly decreased $(P<0.001)$ at first and then decreased $(P>0.05)$ (Figure 3(c)). $t 4$ increased $(P>0.05)$, while $h 4$ decreased $(P>0.05)$ at first and then increased $(P>0.05)$ (Figure 3(d)). Both $w 1$ and $w 2$ demonstrated significant reductions at first $(P<0.01)$ and then had no significant changes $(P>0.05)$ (Figure 3(e)).

3.4. Changes in Pulsographic Parameters during Diastole in PD Patients. As shown in Figure 4, from T0 to T1, time of the peak of dicrotic wave increased $(P>0.05)$ and amplitude of the peak of dicrotic wave $(h(\mathrm{~d}))$ significantly decreased $(P<$ $0.001)$. From $T 1$ to $T 3, t(\mathrm{~d})$ increased $(P>0.05)$, while $h(\mathrm{~d})$ increased significantly $(P<0.001)$ firstly and then increased mildly $(P>0.05)$.

3.5. Changes in Heart Rate in PD Patients. Heart rate was $71.930 \pm 11.22 \mathrm{times} / \mathrm{min}$ at $T 0$ and decreased to $69.63 \pm 6.24$ times/min at $T 1(P>0.05)$. After acupuncture treatment, heart rate significantly decreased to $66.53 \pm 6.94$ times $/ \mathrm{min}$ at T2 $(P<0.01)$ and increased to $67.63 \pm 9.14$ times $/ \mathrm{min}$ at $T 3$ $(P>0.05)$ (Figure 5).

3.6. Changes in Normalized-Based Time Parameters. In view of the fact that time parameters will be effected by different heart rates, in this paper, time parameters of pulsograph at 4 time points were normalized at 75 times/min heart rate and named as $t^{\prime}$. The comparison of $t^{\prime}$ results after normalization is shown in Figure 6; compared with those at $T 0$, there was no difference in $t 1^{\prime}, t 4^{\prime}$, and $t(\mathrm{~d})^{\prime}(P>0.05)$ at $T 1$. Meanwhile, $t 2^{\prime}$ and $t 3^{\prime}$ significantly decreased from $T 0$ to $T 1(P<0.001$, $0.05)$. From $T 1$ to $T 3, t 1^{\prime}$ and $t 4^{\prime}$ significantly decreased at first $(P<0.001)$ and then remained unchanged $(P>0.05)$. And there was no significant change in $t 2^{\prime}, t 3^{\prime}$, and $t(\mathrm{~d})^{\prime}$ from $T 1$ to $T 3(P>0.05)$.

\section{Discussion}

Primary dysmenorrhea (PD) is defined as a cramp-like pain in the lower abdomen before or during menstruation without any identifiable pelvic pathology. Pain may be accompanied by lower back pain, nausea, vomiting, and diarrhea. $\mathrm{PD}$ is frequently found in young nullipara [17]. Modern medical science holds that the emergence of PD is related with many factors. Among them, temporary ischemia of myometrium and endometrium of the uterus plays a part. The ischemia may result from pressured intermuscular blood vessels induced by forceful contraction of uterine arteries and paroxysmal contraction of uterine smooth muscles [18]. 


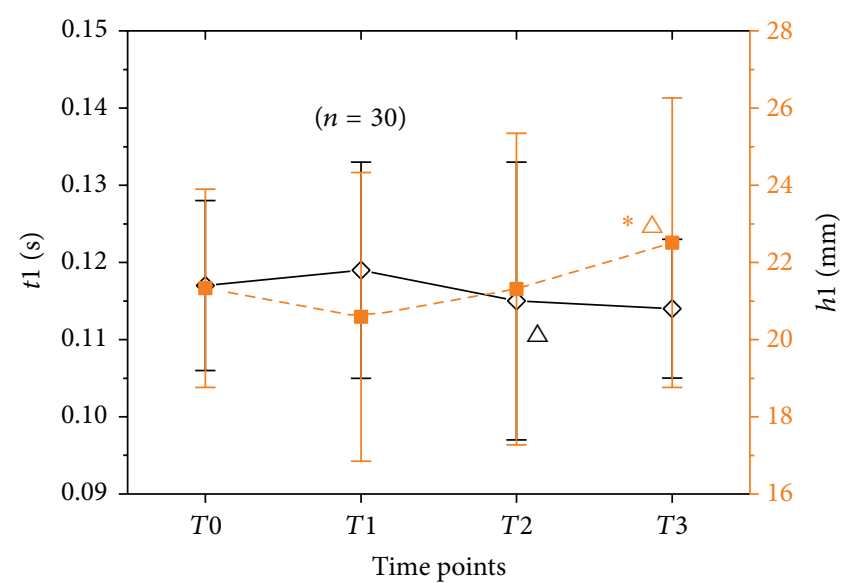

$$
\begin{array}{ll}
\prec & t 1 \\
-- & h 1
\end{array}
$$

(a)

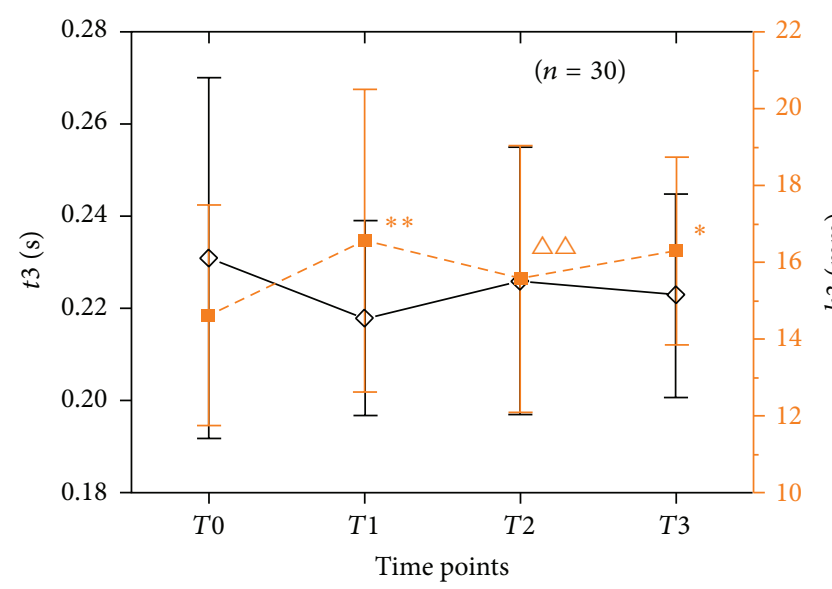$$
\begin{array}{ll}
\multimap & t 3 \\
- & -h 3
\end{array}
$$

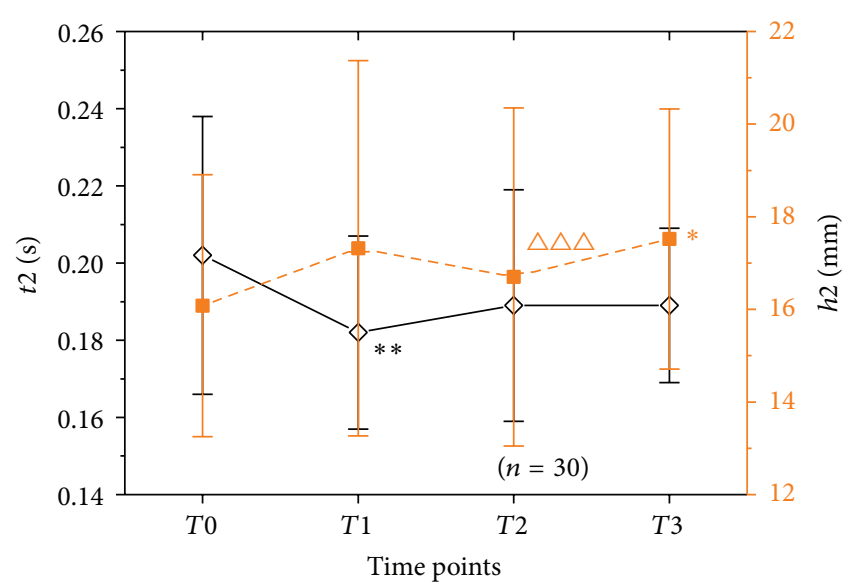

$\diamond t 2$

$--h 2$

(b)

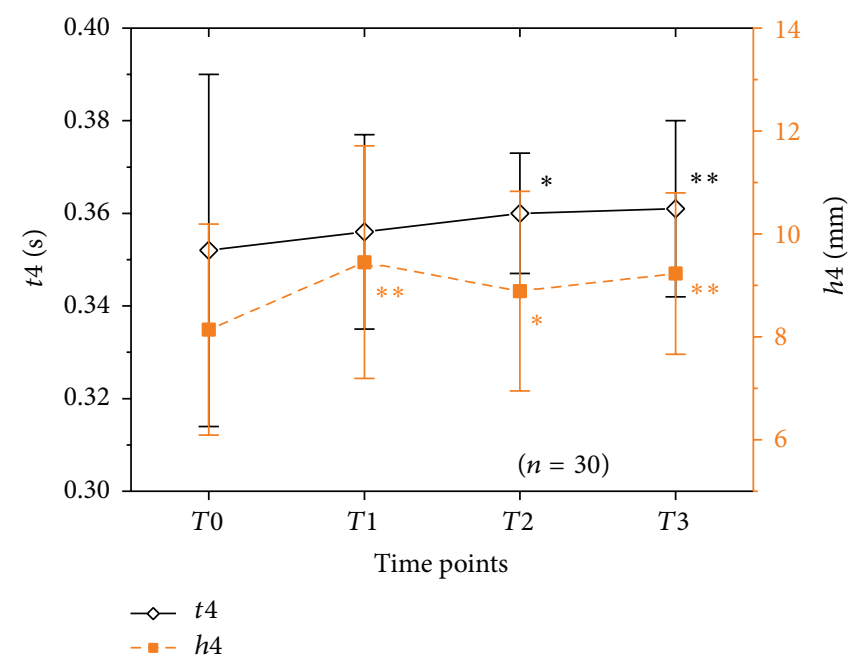

(d)

(c)

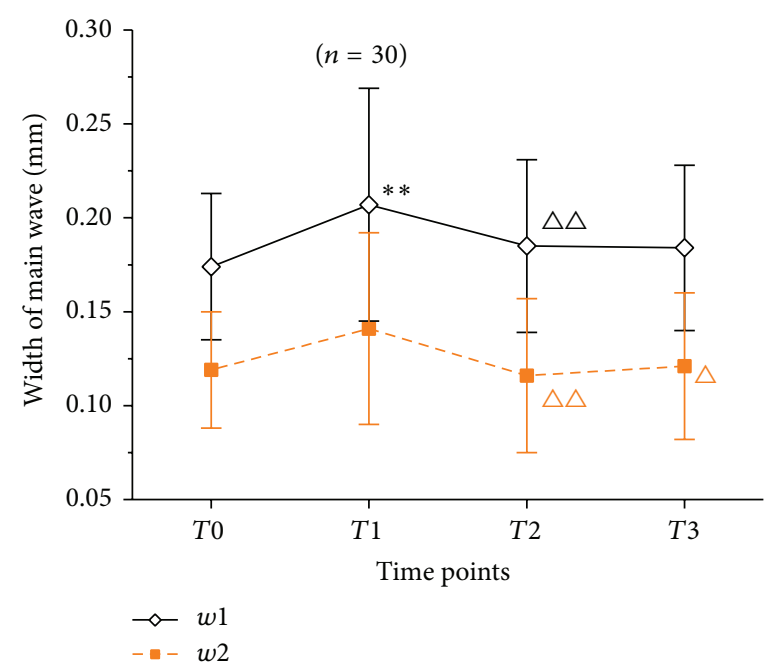

(e)

Figure 3: Pulsograph parameters during systole in 30 PD patients at T0,T1, T2, and T3. Notes: (a), (b), (c), and (d) were the changes in the time and amplitude of D1, D2, D3, and D4. (e) was the changes in the width of the upper $1 / 3(w 1)$ and $1 / 5(w 2)$ of the main wave. ${ }^{* *} P<0.01$ and ${ }^{*} P<0.05$, compared with $T 0 ;{ }^{\triangle} \triangle P<0.001,{ }^{\triangle} \triangle P<0.01$, and ${ }^{\triangle} P<0.05$, compared with $T 1$. 


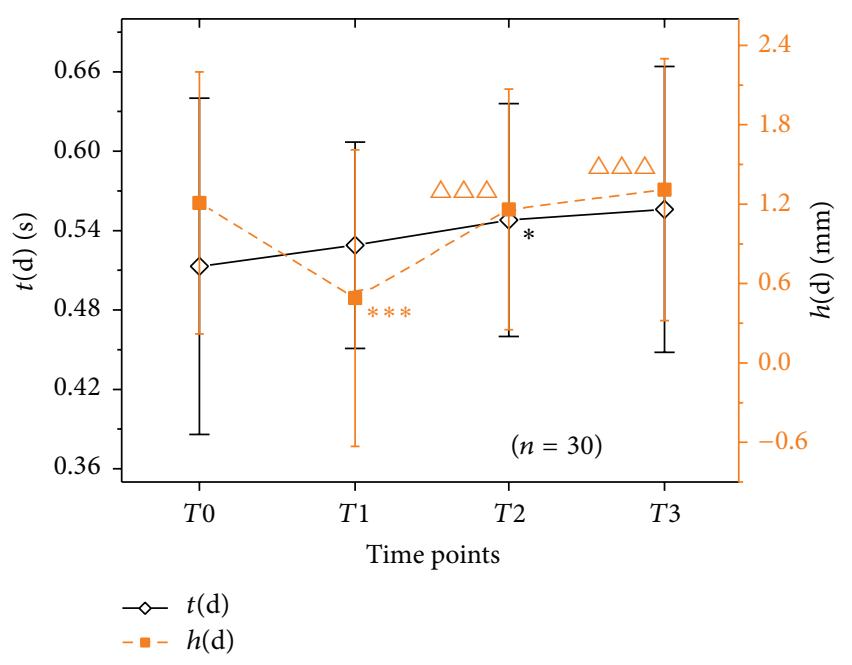

FIGURE 4: Pulsographic parameters during diastole in $30 \mathrm{PD}$ patients at T0,T1, T2, and T3. Notes: ${ }^{* * *} P<0.001$ and ${ }^{*} P<0.05$, compared with $T 0 ;{ }^{\triangle \triangle} P<0.001$, compared with $T 1$.

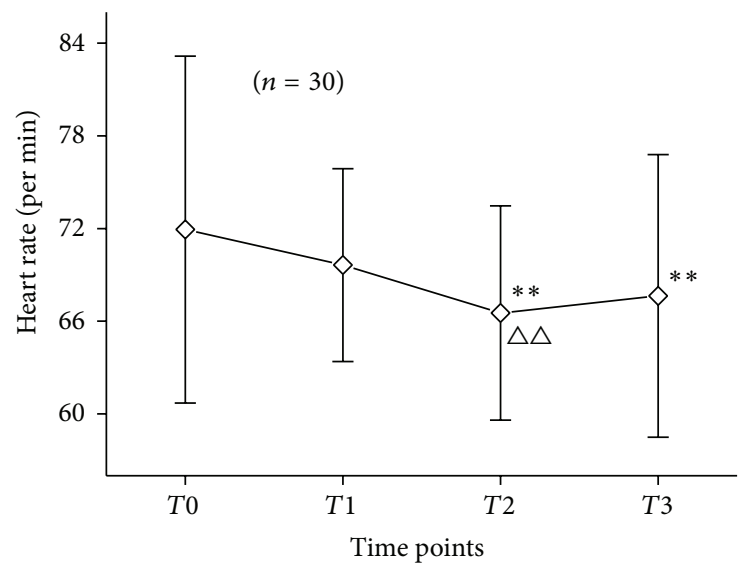

Figure 5: Changes in heart rate in $30 \mathrm{PD}$ patients at $T 0, T 1, T 2$, and T3. Notes: ${ }^{* *} P<0.01$, compared with $T 0 ;{ }^{\triangle} P<0.01$, compared with $T 1$.

Traditional Chinese Medicine holds that PD is often caused by blood stasis blocking the uterus. That is to say, during menstruation, $q i$ and blood fail to flow freely. Pain arises as a result. Apart from pain, changes in movement of $q i$ and blood would also affect cunkou pulse [19].

In this study, pulsographic parameters of cunkou pulse before dysmenorrhea and in obvious dysmenorrhea and after dysmenorrheal relieving in PD patients were investigated. Considering effect of different heart rates on the time parameters of pulsograph, in this experiment, the time parameters were normalized at the heart rate of 75 times/min. In so doing, we were more inclined to think that normalized time parameters could better reflect the effect of pain on time parameters in PD patients.

Changes in pulsographic parameters revealed almost opposite changing trends from before dysmenorrhea to before dysmenorrhea (from $T 0$ to $T 1$ ) and finally to after dysmenorrheal relieving (from $T 1$ to $T 2$ ). Although the effect of menstruation on pulsographic parameters cannot be excluded from $T 0$ to $T 1$, combining with the changes from $T 1$ to $T 2$ we can come to a preliminary conclusion that pain could cause increases in width of the upper $1 / 3$ of main wave $(w 1)$, in amplitude of gorge of main wave $(h 2)$, and in amplitude of dicrotic notch (h4) and decreases in time of gorge of main wave ( $t 2)$ and in amplitude of peak of dicrotic wave $(h(\mathrm{~d}))$.

Interestingly, we also noticed different changing trends from the commencement of acupuncture to 30 mins after acupuncture (from $T 1$ to $T 2$ and from $T 2$ to $T 3$ ). Part of parameters showed continuous similar changes. For example, $t 1$ kept decreasing and $h 1, t 4, t(\mathrm{~d})$, and $h(\mathrm{~d})$ kept increasing. Some changes from $T 1$ to $T 2$ and from $T 2$ to $T 3$ were in opposite directions. For instance, $h 3$ and $h 4$ increased at first and then decreased, and $t 3$ decreased at first and then increased. Still others changed significantly from $T 1$ to $T 2$ but no obvious changes from $T 2$ to $T 3$ were observed, such as $w 1$ and $w 2$, which decreased at first and then almost did not change. The possible reason for this phenomenon may be related to the negative feedback regulation which caused corresponding changes in the radial artery.

Previous researches show that each pulse is of specific pulsograph features. The changes in pulsographic parameters in our research could be interpreted with reference to wiry pulse, slippery pulse, and forceful pulse. The pulsograph of wiry pulse is characterized by higher prodicrotic wave, close to or fused to the main wave, manifesting as a broad single peak main wave, and higher dicrotic notch, and flat dicrotic wave. The pulsograph of slippery pulse is characterized by a higher double-humped main wave with big-slope ascending and descending branches, and a delayed prodicrotic wave, and a higher dicrotic wave. In addition, pulsograph of forceful pulse is characterized by a higher main wave [20]. In this paper, the pulsographs of patients with obvious pain manifested as wider upper $1 / 3$ of main wave, and earlier gorge of main wave and higher prodicrotic wave, and higher dicrotic notch, and lower dicrotic wave, compared with pulsographs of those without pain. All these changes showed that pulsographs of patients with obvious pain resembled that of wiry pulse. However, when the pain is relieved, the above parameters change to the other way around. As the gorge of main wave gets higher and earlier, the dicrotic notch comes earlier while the time of dicrotic wave does not change obviously, which indicated that the slope of ascending and descending branches is elevated and there is a trend toward moving to a slippery pulse with a weakened pulse-power. This finding was consistent with the records in TCM that pain is often associated with wiry pulse.

As a pilot study we have to admit that there are some limitations in this study. Firstly, a limitation was the small number of enrolled patients and lack of control group. Without control group of healthy females, influence of menstruation on pulsograph could not be excluded. Secondly, the pain of PD cannot represent all kinds of pain owing to its localization and patient population particularity. Further studies on different types of pain influencing pulsograph are 


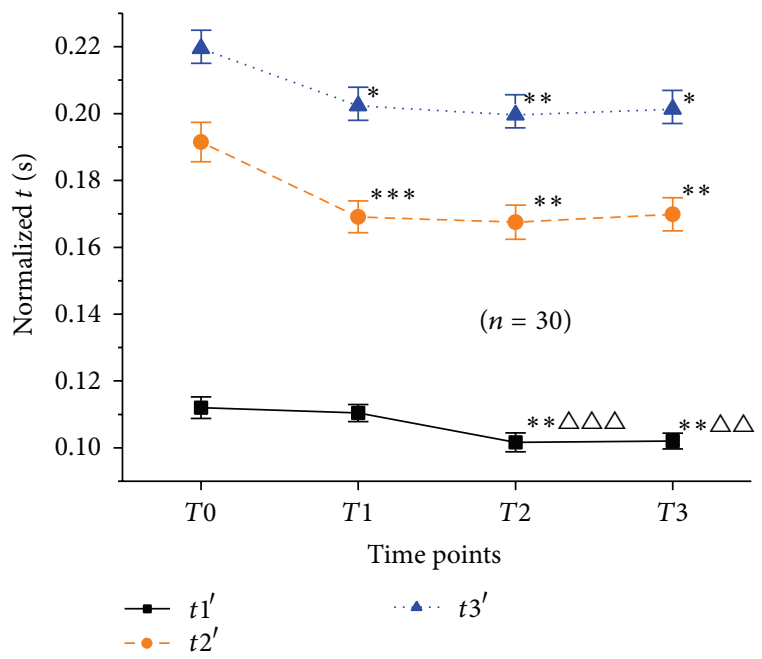

(a)

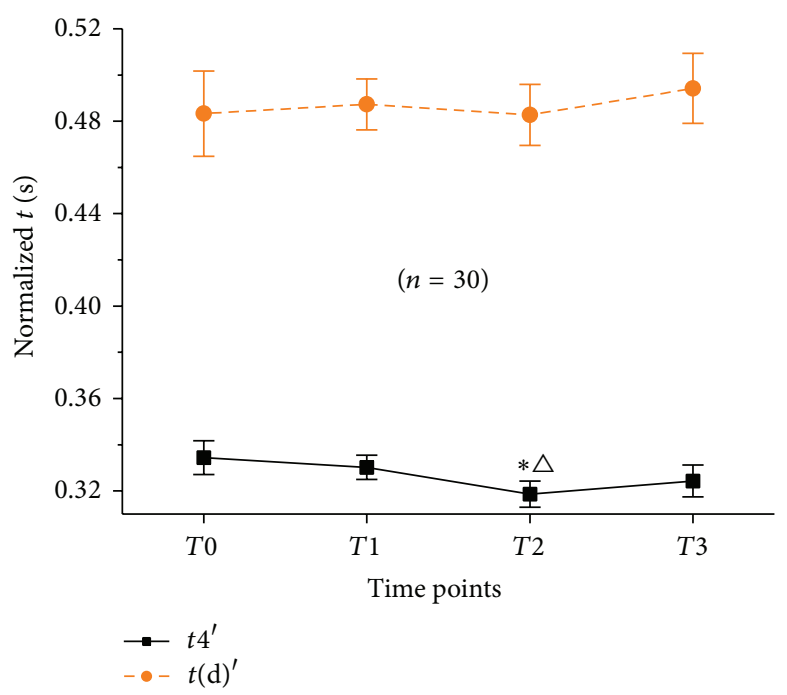

(b)

FIGURE 6: Changes in normalized-based time parameters at T0, T1, T2, and T3. Notes: (a) was changes in $t 1^{\prime}, t 2^{\prime}$, and $t 3^{\prime}$; (b) was changes in $t 4^{\prime}$ and $t(\mathrm{~d})^{\prime} .{ }^{* * *} P<0.001,{ }^{* *} P<0.01$, and ${ }^{*} P<0.05$, compared with $T 0 ;{ }^{\triangle \triangle} P<0.001,{ }^{\triangle \triangle} P<0.01$, and ${ }^{\triangle} P<0.05$, compared with $T 1$.

needed before we can know for sure how pain affects pulsographic parameters.

\section{Conclusions}

There are changes in pulsographic parameters, basically in opposite trends, in patients with PD when their pain occurs and when it is relieved. Further studies with control groups are needed to determine whether pulsographic parameters can be used as an objective indicator for pain changes in patients with primary dysmenorrhea.

\section{Conflict of Interests}

The authors declare that there is no conflict of interests regarding the publication of this paper.

\section{Acknowledgments}

This work was sponsored by the National Natural Science Foundation of China (no. 81473598), Self-Research of Beijing University of Chinese Medicine (no. 2014-JYBZZ-XS-011), Project of Teaching Masters of Beijing Educational Committee "Research and Implementation of Bilingual Education," Reform and Research in Teaching of Outstanding Innovation Team of Beijing High Schools' Continuing Education (Diagnostics of Traditional Chinese Medicine), Innovation Team of Beijing University of Chinese Medicine (no. 2011-CXTD-08), and 111 Project of China Ministry of Education (no. B07007).

\section{References}

[1] S. Walsh and E. King, Pulse Diagnosis: A Clinical Guide, Churchill Livingstone, Edinburgh, Scotland, 2008.
[2] S. L. Ji and Z. Z. Cheng, Diagnostics of Traditional Chinese Medicine, People's Medical Publishing House, Beijing, China, 2006.

[3] L. J. Wang, "Preliminarily study of the relationship between pain and wiry pulse - analysis of 280 cases," Xinjiang Journal of Traditional Chinese Medicine, vol. 1, pp. 17-19, 1987.

[4] X. Q. Xiao, Y. Q. Wang, H. X. Yan et al., "Objectification of Chinese pulse diagnosis," Chinese Archives of Traditional Chinese Medicine, pp. 2042-2204, 2011.

[5] H. J. Wu, Z. F. Zhang, J. T. Xu et al., "Relationship between body surfacearea, BMI and taking pulse pressure, pulsograph parameters in 677 healthy persons," China of Traditional Chinese Medicine and Pharmacy, vol. 5, pp. 1361-1365, 2013.

[6] R. Li, L. Yong, C. Y. Liu et al., "Pulse feature and change rule of pulsograph parameters in deficiency and cold patterns," Acta Universitatis Traditionis Medicalis Sinensis Pharmacologiaeque Shanghai, vol. 1, pp. 37-39, 2007.

[7] J. B. Tai, D. Z. Zhu, and C. Q. Ling, "Comparative research on pulsographs of qi deficiency and blood stasis in primary liver cancer patients," Chinese Archives of Traditional Chinese Medicine, vol. 1, pp. 118-122, 2011.

[8] Y. Zhao, L. M. Ou-Yang, and F. Zeng, "Correlational analyses of the syndrome differentiation and pulsographic parameters in primary dysmenorrheal," Shangdong Journal of Traditional Chinese Medicine, vol. 11, pp. 743-744, 2007.

[9] Y. Zhao and X. J. Sun, "Pulsograph analysis of deficiency and excess patterns in primary dysmenorrheal," Hebei Traditional Chinese Medicine, vol. 3, pp. 350-351, 2009.

[10] G. Lefebvre, O. Pinsonneault, and V. Antao, "Primary dysmenorrheal consensus guideline," SOGC Clinical Practice Guidelines, vol. 27, no. 12, pp. 1117-1130, 2005.

[11] G. X. Shi, Q. Q. Li, C. Z. Liu et al., "Effect of acupuncture on deqi traits and pain intensity in primary dysmenorrheal: analysis of data from a larger randomized controlled trial," BMC Complementary and Alternative Medicine, vol. 14, article 69, 2014.

[12] D. Di, M. Zhou, P. F. Qin et al., "Developments acquisition of Chinese medicine pulse research," Shanghai Journal of Traditional Chinese Medicine, vol. 7, pp. 104-108, 2014. 
[13] D. Y. Zhu, The immediate analgesia effect observation of acupuncturing Diji and acupuncturing Diji and Guanyuan to cure patients with primary dysmenorrheal [Ph.D. thesis], Shandong University of Traditional Chinese Medicine, Jinan, China, 2014.

[14] J. Y. Hou, "Clinical on acupuncture treatment in patients with primary dysmenorrheal," Guangming Journal of Chinese Medicine, vol. 2, pp. 330-332, 2010.

[15] M. L. Proctor and P. A. Murphy, "Herbal and dietary therapies for primary and secondary dysmenorrheal," Cochrane Database of Systematic Reviews, no. 3, Article ID CD002124, 2001.

[16] D. Z. Ye, "Describing of the rules of selecting points in treatment of primary dysmenorrheal with acupuncture," Chinese and Foreign Medical Research, vol. 10, pp. 147-149, 2015.

[17] X. Xie and W. L. Gou, Obstetrics and Gynecology, 8 Versions, People's Medical Publishing House, Beijing, China, 2013.

[18] D. Chung and R. L. Caruso, "Potential role for oxidative stress in $2,2^{\prime}$-dichlorobiphenyl-induced inhibition of uterine contractions but not myometrial gap junctions," Toxicological Sciences, vol. 93, no. 1, pp. 172-179, 2006.

[19] Y. Z. Zhang, Gynecology of Traditional Chinese Medicine, China Traditional Medicine Press, Beijing, China, 2007.

[20] Z. F. Fei, Modern Pulse Diagnosis in Traditional Chinese Medicine, People's Medical Publishing House, Beijing, China, 2003. 


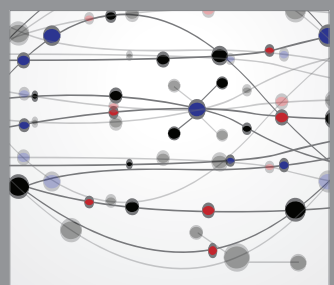

The Scientific World Journal
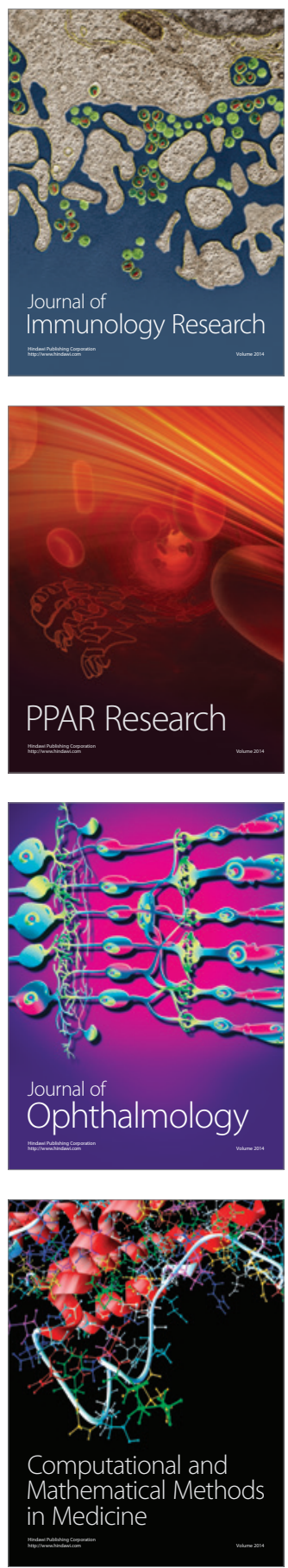

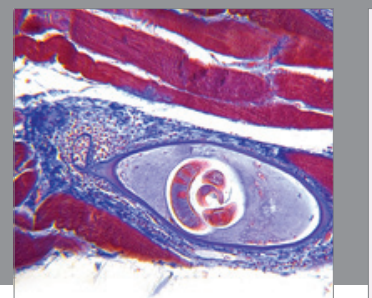

Gastroenterology

Research and Practice
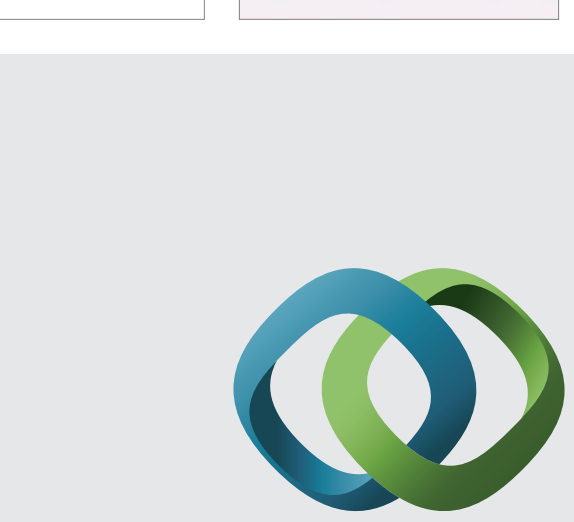

\section{Hindawi}

Submit your manuscripts at

http://www.hindawi.com
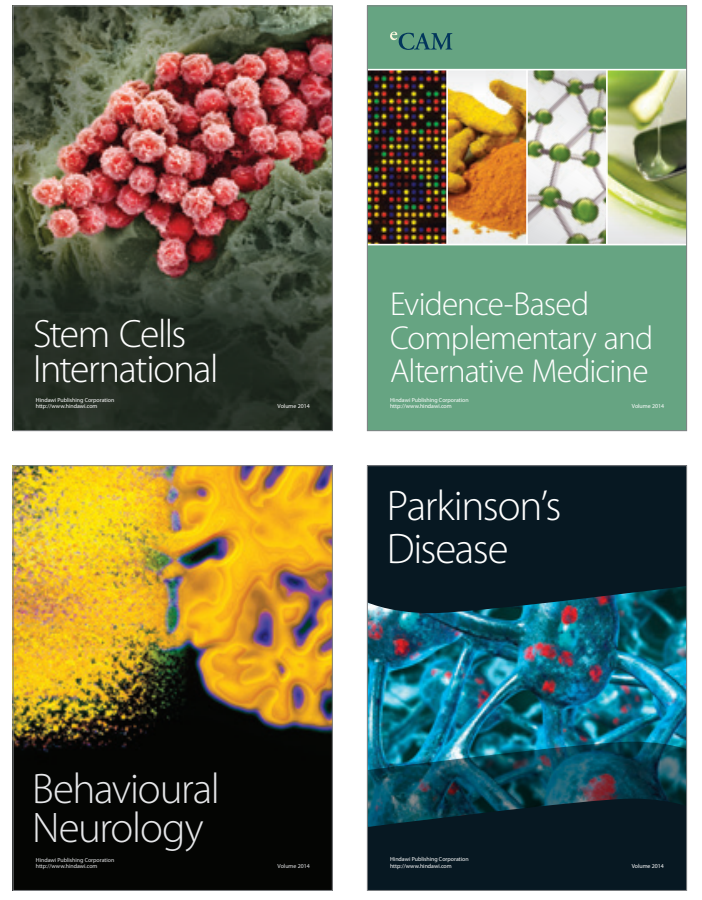
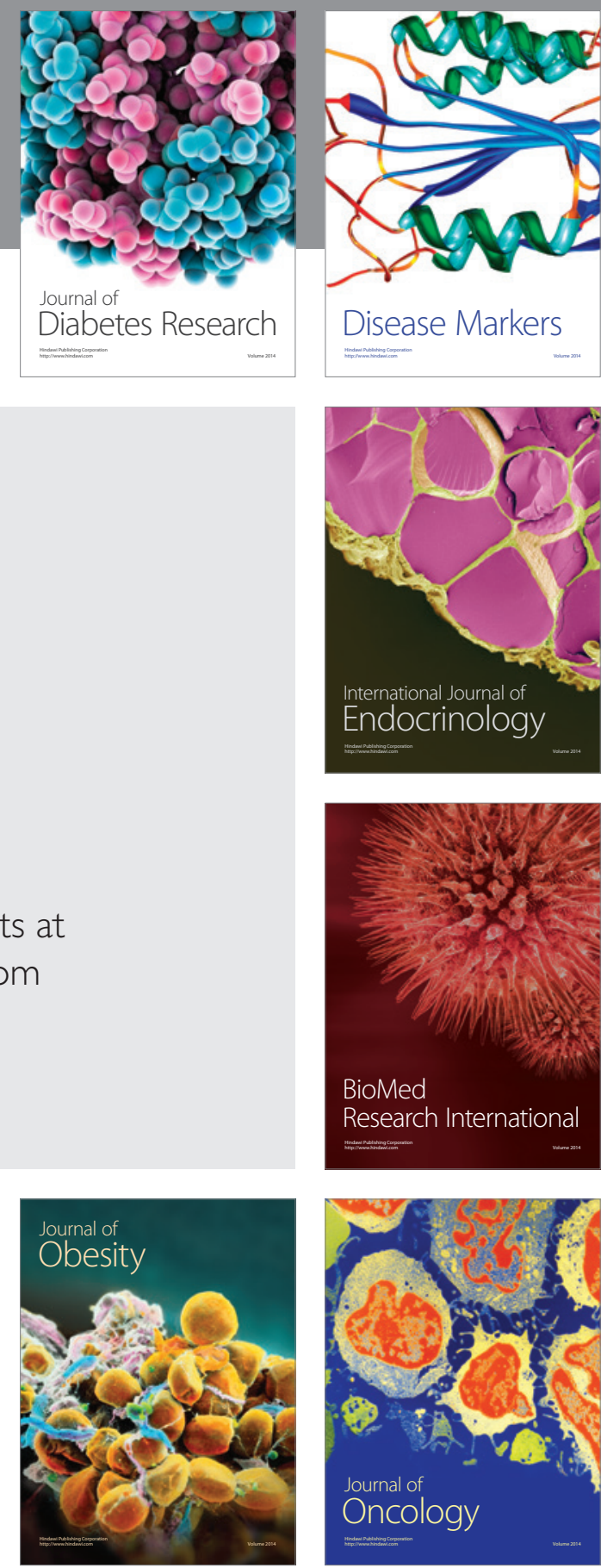

Disease Markers
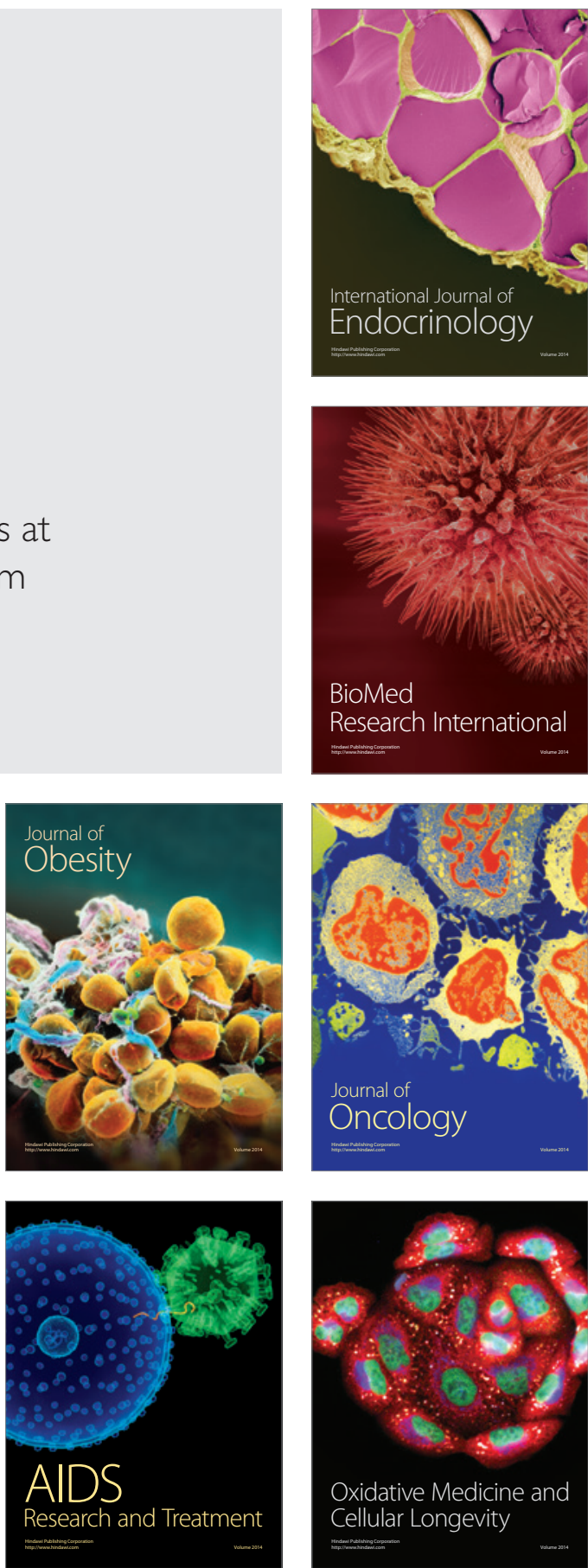